\title{
Dense plasma interferometry with a tabletop soft X-ray laser and an amplitude division interferometer based on diffraction gratings
}

\author{
J. Filevich ${ }^{a}$, M.C. Marconi ${ }^{a}$, K. Kanizay, J.L.A. Chilla and J.J. Rocca \\ Department of Electrical and Computer Engineering, Colorado State University, Fort Collins, \\ CO 80523, U.S.A.
}

\begin{abstract}
We have demonstrated a novel Mach-Zehnder soft $\mathrm{x}$-ray interferometer that uses diffraction gratings as beam splitters. The interferometer was used together with a $46.9 \mathrm{~nm}$ tabletop soft $\mathrm{x}$-ray laser, to map the evolution of the electron density distribution of a large-scale laser created plasma.
\end{abstract}

\section{INTRODUCTION}

The development of gain-saturated soft $x$-ray lasers has opened the possibility to extend laser interferometry to large scale plasmas of very high density that can not be probed with optical lasers, and has generated interest in soft $x$-ray laser interferometers [1-3]. The recent advent of gain-saturated tabletop soft $x$-ray lascrs creates the opportunity of developing portable soft $x$-ray tools that will allow ictailed maps of the electron density evolution in a great variety of very dense plasmas. Recently our group has demonstrated plasma interferometry combining a $46.9 \mathrm{~nm}$ capillary discharge tabletop laser with a wavefront-division interferometer based on Lloyd's mirror [3]. The Lloyd's mirror has the advantage of constituting the simplest possible soft $\mathrm{x}$-ray interferometer. However, it also presents some limitations: since this is a wavefront-division interferometer in which the fringe visibility relays in the spatial and temporal coherence of the laser, it is difficult to obtain good fringe visibility over a

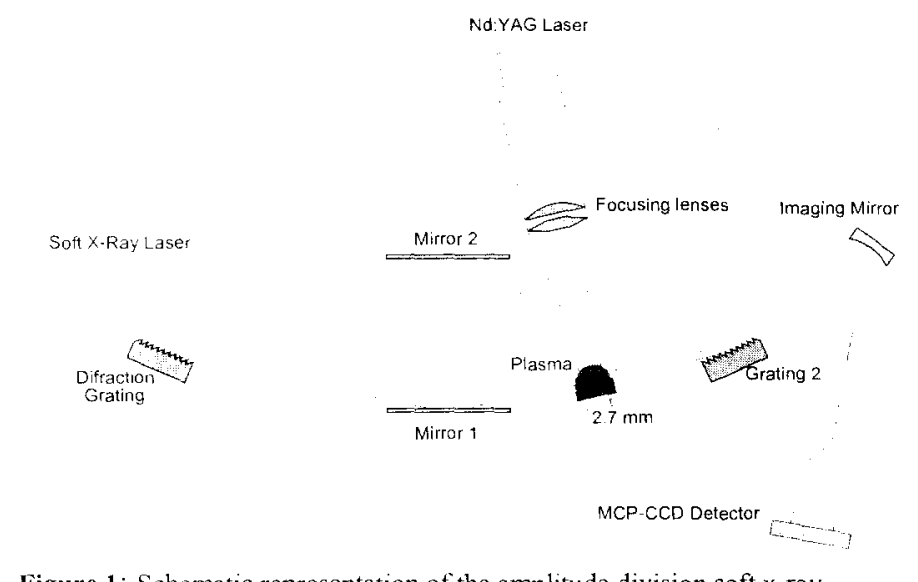

Figure 1: Schematic representation of the amplitude division soft $x$-ray interferometer based on diffraction gratings.

large area. To overcome this limitation we have developed a novel soft $\mathrm{x}$-ray amplitude division interferometer in which diffraction gratings are used as beam splitters. By properly tailoring the

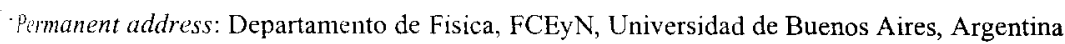




\title{
Dense plasma interferometry with a tabletop soft X-ray laser and an amplitude division interferometer based on diffraction gratings
}

\author{
J. Filevich ${ }^{a}$, M.C. Marconi ${ }^{a}$, K. Kanizay, J.L.A. Chilla and J.J. Rocca \\ Department of Electrical and Computer Engineering, Colorado State University, Fort Collins, \\ CO 80523, U.S.A.
}

\begin{abstract}
We have demonstrated a novel Mach-Zehnder soft x-ray interferometer that uses diffraction gratings as beam splittess. The interferometer was used together with a $46.9 \mathrm{~nm}$ tabletop soft x-ray laser, to map the evolution of the electron density distribution of a large-scale laser created plasma.
\end{abstract}

\section{INTRODUCTION}

The development of gain-saturated soft $x$-ray lasers has opened the possibility to extend laser interferometry to large scale plasmas of very high density that can not be probed with optical lasers, and has generated interest in soft $x$-ray laser interferometers [1-3]. The recent advent of gain-saturated tabletop soft $x$-ray lasers creates the opportunity of developing portable soft $x$-ray tools that will allow detailed maps of the electron density evolution in a great variety of very dense plasmas. Recently our group has demonstrated plasma interferometry combining a $46.9 \mathrm{~nm}$ capillary discharge tabletop laser with a wavefront-division interferometer based on Lloyd's mirror [3]. The Lloyd's mirror has the advantage of constituting the simplest possible soft $\mathrm{x}$-ray interferometer. However, it also presents some limitations: since this is a wavefront-division interferometer in which the fringe visibility relays on the spatial and temporal coherence of the laser, it is difficult to obtain good fringe visibility over a

Nd:YAG Laser

Soft X-Ray Laser

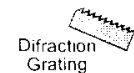

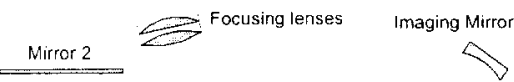

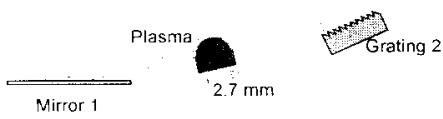

MCP-CCD Detector

Figure 1: Schematic representation of the amplitude division soft $x$-ray interferometer based on diffraction gratings

large area. To overcome this limitation we have developed a novel soft $\mathrm{x}$-ray amplitude division interferometer in which diffraction gratings are used as beam splitters. By properly tailoring the

a Permanent address: Departamento de Fisica, FCEyN, Universidad de Buenos Aires, Argentina. 
gratings, the interferometer can be made to operate at any selected soft $x$-ray wavelength. The interferometer was used in combination with a $46.9 \mathrm{~nm}$ capillary discharge tabletop soft $\mathrm{x}$-ray laser [4] to map the electron density evolution of a $2.7 \mathrm{~mm}$ long line focused plasma.

\section{SOFT X-RAY INTERFEROMETER BASED ON DIFFRACTION GRATINGS}

The soft $x$-ray interferometer, which consists of a modified Mach-Zehnder configuration [7], is schematically illustrated in figure 1 . The soft $x$-ray laser beam is diffracted off the first grating into the two arms of the interferometer. The incidence angle and the blaze angle of the grating are selected to allow for most of the laser radiation to be evenly split between the zero and first diffraction orders. This is essential for obtaining a good fringe visibility. Two elongated grazing incidence mirrors redirect the zero and first order arms towards the second grating, which recombines the beams to generate the interference pattern. By tilting the grazing incidence mirrors and the output grating, the fringe spacing

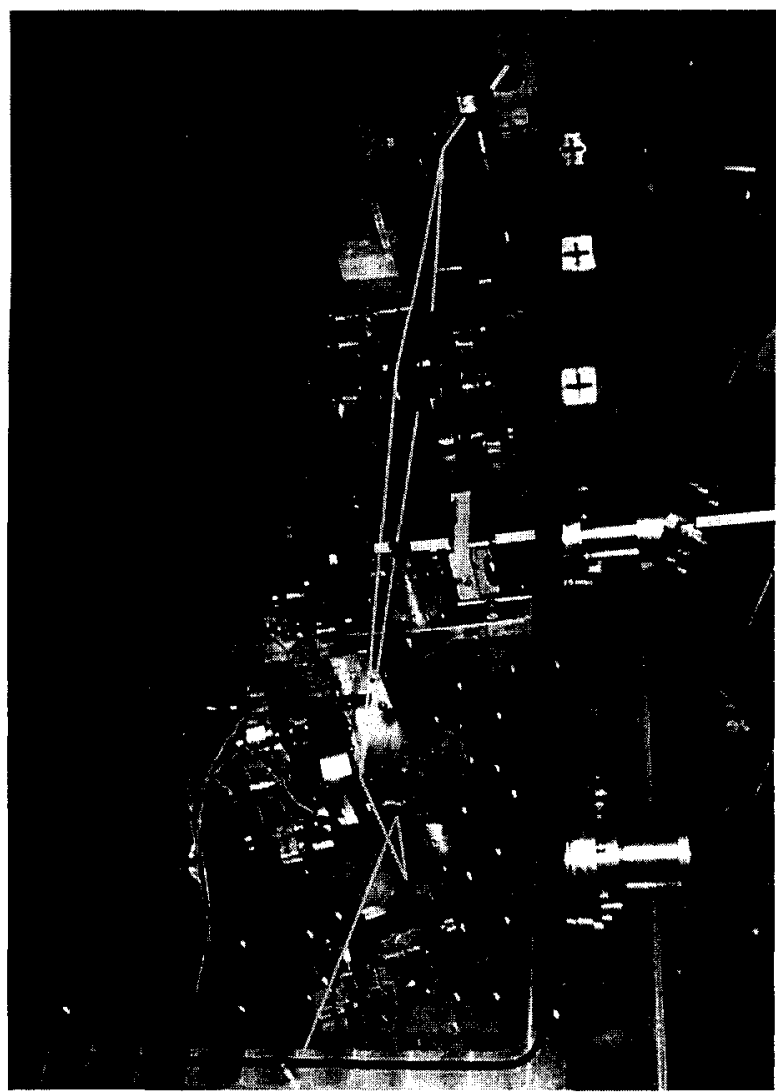

Figure 2: Amplitude division soft $x$-ray interferometer, inside its vacuum chamber. The lines represent the path of the soft $x$-ray (thin lines) laser and the Nd:YAG laser beam(thick line).

and orientation can be adjusted. A Si/Sc multilayer mirror [5] of 30cm focal length and $\approx 40 \%$ reflectivity images the plasma with a magnification of about $25 \times$ onto a MCP-CCD detector. A flat mirror (not shown in fig.1) was used to relay the image. The interferometer was prealigned using an infrared $(824 \mathrm{~nm})$ semiconductor laser diode selected to have a temporal coherence length of $\sim 250 \mu \mathrm{m}$, similar to that of the soft $x$-ray laser. To allow the alignment of the interferometer with the diode laser the gratings were designed with two vertically separated rulings on the same substrate. Part of the substrate was ruled with $300 \mathrm{l} / \mathrm{mm}$ to diffract the $46.9 \mathrm{~nm}$ laser beam, and the other with $17.06 \mathrm{l} / \mathrm{mm}$ to diffract the beam of the alignment diode. With this ratio of groove spacing we obtain the same dispersion and therefore the sam diode. All the relevant movemen the system was under vacuum. In entire field of view. The interferor

\section{INTERFEROMETRY OF I}

We studied a plasma generated $\mathrm{b}$ wavelength of $1.06 \mu \mathrm{m}$ into a $\sim 30$ on one of the arms of the interf plasma created with $0.36 \mathrm{~J} \mathrm{Nd-YA}$ generated by amplification of the
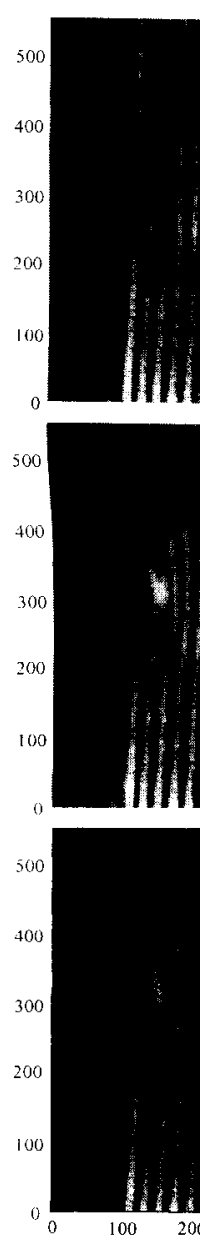

Figure 3: Sequen $2.7 \mathrm{~mm}$ long line $\mathrm{f}$ respect to the begir

discharge current pulse. This tat FWHM duration [4]. The far fiel about $4.6 \mathrm{mrad}$. 
th. The laser [4]

$1[7]$, is into the lected to ers. This irect the rate the spacing

$\approx 40 \%$ r. A flat using an $250 \mu \mathrm{m}$, de laser it of the l/mm 10 he same dispersion and therefore the same path for the beams of the soft $x$-ray laser and the alignment laser diode. All the relevant movements in the system were motorized to fine-tune the interferometer once the system was under vacuum. Interferograms with very good visibility $(\sim 0.5)$ were obtained over the entire field of view. The interferometer showed excellent stability.

\section{INTERFEROMETRY OF LASER CREATED PLASMA}

We studied a plasma generated by focusing a Q-switched Nd-YAG laser operating at its fundamental wavelength of $1.06 \mu \mathrm{m}$ into a $\sim 30 \mu \mathrm{m}$ wide line focus $\sim 2.7 \mathrm{~mm}$ in length, onto a Cu slab target, placed on one of the arms of the interferometer. The results discussed below correspond to the study of a plasma created with $0.36 \mathrm{~J} \mathrm{Nd-YAG} \mathrm{laser} \mathrm{pulses} \mathrm{of} \sim 13 \mathrm{~ns}$ FWHM duration. The probe laser pulses were generated by amplification of the $46.9 \mathrm{~nm}$ line of Ne-like Ar in an Ar capillary plasma excited by a fast
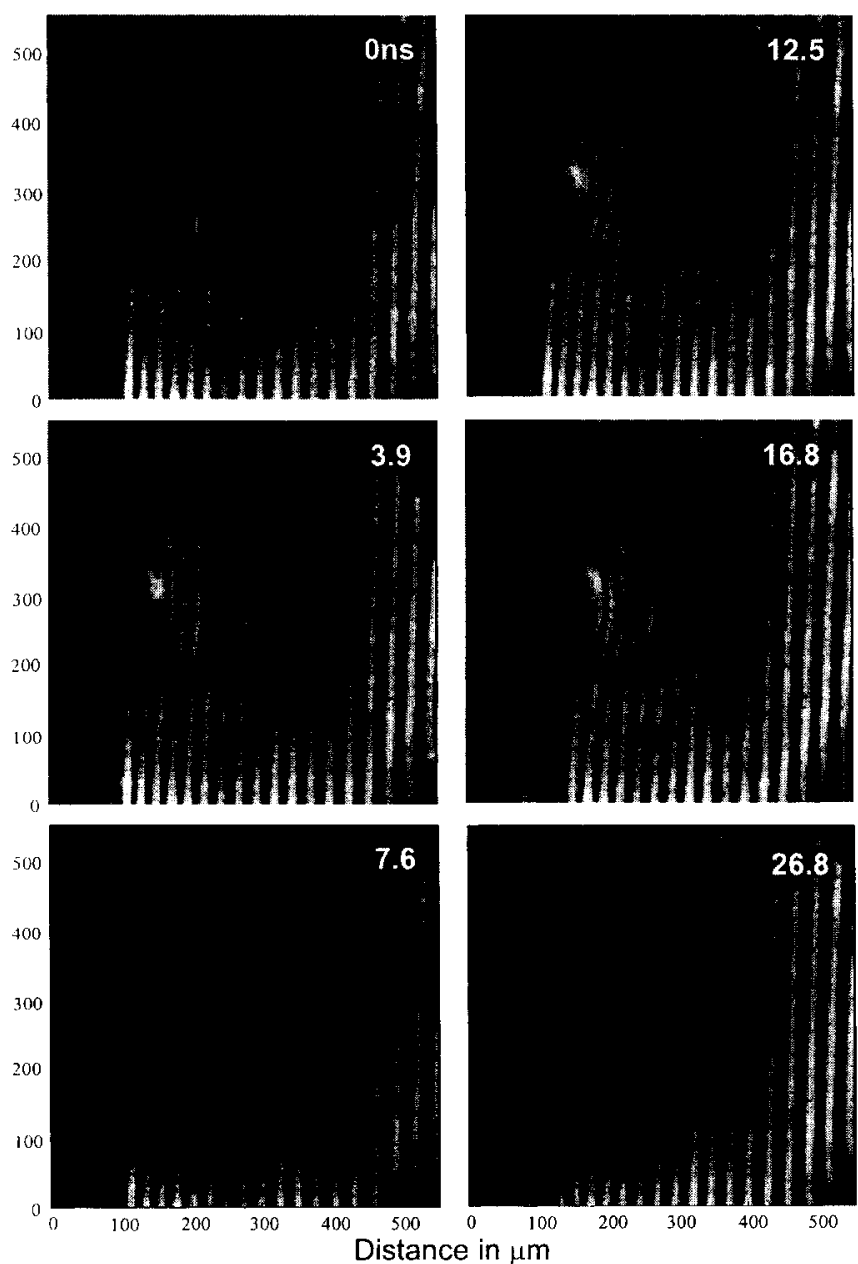

Figure 3: Sequence of soft $x$-ray interferograms depicting the evolution of a $2.7 \mathrm{~mm}$ long line focused plasma generated by a Nd:YAG laser. The time delay respect to the beginning of the laser pulse is indicated in each interferogram.

discharge current pulse. This tabletop laser produces pulses with energy of about $0.13 \mathrm{~mJ}$ and $1.2 \mathrm{~ns}$ FWHM duration [4]. The far field beam profile has an annular shape and a peak to peak divergence of about $4.6 \mathrm{mrad}$. 
The sequence of interferograms shown on figure 3 describes the evolution of the laser-created plasma The fringes are observed to shift with time due to the presence of the plasma. At the latter times the plasma cools down and absorption of the probe beam by the plasma becomes noticeable. Electron density maps were computed from the measured number of fringe shifts at each location assuming the plasma is uniform along the line focus. We have been able to probe the plasma at locations as close as $25-30 \mu \mathrm{m}$ from the target, where the electron density is $\sim 5 \times 10^{19} \mathrm{~cm}^{-3}$ and the density gradient is steep. Ray tracing computations show that this measurement would be difficult to realize with an UV laser owing to the strong refraction caused by the large density gradient. Another series of measurements for $1.8 \mathrm{~mm}$ long line-focused plasmas created by $0.6 \mathrm{~J} \mathrm{Nd}$ :YAG laser pulses probed electron densities as great as $1 \times 10^{20} \mathrm{~cm}^{-3}[6]$.

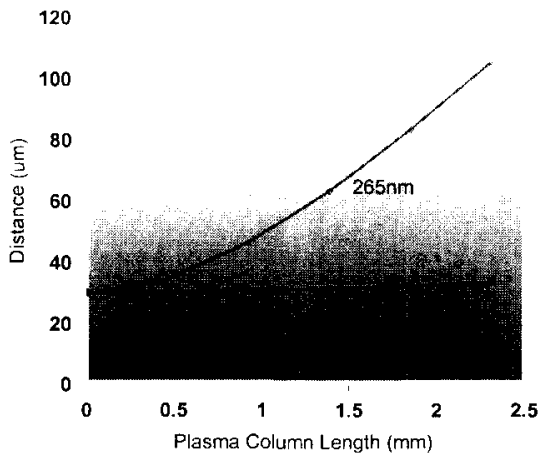

Figure4: Computed trajectory of rays corresponding to a $46.9 \mathrm{~nm}$ (Ne-like Ar laser) and $265 \mathrm{~nm}$ $\left(4^{\text {th }}\right.$ harmonic of $\left.\mathrm{Nd}: \mathrm{YAG}\right)$ traversing a plasma with electron density distribution corresponding to the $12.5 \mathrm{~ns}$ interferogram in figure 3

\section{CONCIUSIONS}

We have demonstrated a new amplitude division interferometer utilizing diffraction gratings as beam splitters. It was used in combination with a $46.9 \mathrm{~nm}$ capillary discharge laser to map the electron density evolution of a large-scale laser created plasma. This interferometer scheme has the advantage of an increased resistance to plasma debris as compared with amplitude-division interferometers based on thin film beam splitters. This grating interferometer can be combined with any of the presently available saturated soft $x$-ray lasers to study high density laboratory plasmas of significant current interest.

This work was supported by the U.S. Department of Energy grant DE-FG03-98DP00208. We gratefully acknowledge a grant from the W.M.Keck Foundation.

\section{References}

[1] L.B. Da Silva, T.W. Barbee Jr., R. Cauble, P. Celliers, D. Ciarlo, S. Libby, R.A. London, D. Matthews, S Mrowka, J.C. Moreno, D. Ress, J.E. Trebes, A.S. Wan, and F. Weber. Phys. Rev. Lett. 74, 3991, (1995) pp. $3991-3994$

[2] F. Albert, D. Joyeux, P. Jaegle, A. Carillon, J.P. Chauvineau, G. Jamelot, A. Klisnick, J.C. Lagron, D Phalippou, D. Ros, S. Sebban, P. Zeitoun Opt. Comm., 142, 184, (1997) pp. 184-188

[3] C.H. Moreno, M.C. Marconi, K. Kanizay, J.J. Rocca, Yu. A. Unspenskii, A.V. Vinogradov and Yu.A Pershin. Phys. Rev. E; 60, (1999) pp. 911-917

[4| B.R. Benware, C.D. Macchietto, C.H. Moreno, J.J. Rocca. Phys. Rev. Lett. 81, (1998) pp. 5804-5807

[5] Yu. A. Unspenskii, V.A. Lavashov, A.V. Vinogradov, A. I. Fedorenko, V.V. Kondratenko, Yu.P. Pershin, E.N. Zubarev, and V. Yu Fedotov.. Opt. Lett. 23, (1998) pp. 771-773

[6] J. Filevich, K. Kanizay, M. C. Marconi, J. L. A. Chilla, and J. J. Rocca, Opt. Lett. 25, 5, (2000) pp. 356-359

[77 J.L.A. Chilla, J.J. Rocca, O.E. Martinez and M.C. Marconi. Opt Lett 21, 13, (1996) pp. 955-957
Progress in FWSD

\section{S.J. Topping, R. Keenan,} S. Dobosz, G.J. Tallents ${ }^{1}$,

School of Mathematics and Pr Department of Physics, Univ

${ }^{2}$ Rutherford Appleton Laborat

\begin{abstract}
Progress of a fourradiation to shorter waveleng experiment, involving the $\mathrm{Ne}$ $\left(\lambda_{O P !}=1.054 \mu \mathrm{m}\right)$ in a $\mathrm{Na}-\mathrm{lik}$ from 5 optical beams has routi and information from fringe $v i$ XRL. Characterisation of the $g$ interferometry.
\end{abstract}

\section{INTRODUCTION}

A preliminary experimental invest area east of the Vulcan laser at $t$ essentially involves the combinat corresponding to the sum differenc two XRL photons at $231.2 \AA$ from with the aim to achieve a near fr modelling of the third order non-lir of the Vulcan laser may provide u future experiment.

\section{EXPERIMENT}

The Ne-like Ni XRL was created $u$ drive beams had a standard 80 ps distributed across a $100 \mu \mathrm{m} \times 35 \mathrm{~mm}$ pulse was set 2 ns ahead of the $m$ length to simulate a travelling wave

The main diagnostic for the $1200 \mathrm{lines} / \mathrm{mm}$ aperiodic ruled grat primary XRL beam and was later uniformity and the ionisation balan and Bragg spectrometer respectivel mirror and an XRM used to recomb see Figure 5 (a).

The sixth Vulcan beam wa process. This minimised timing prol 